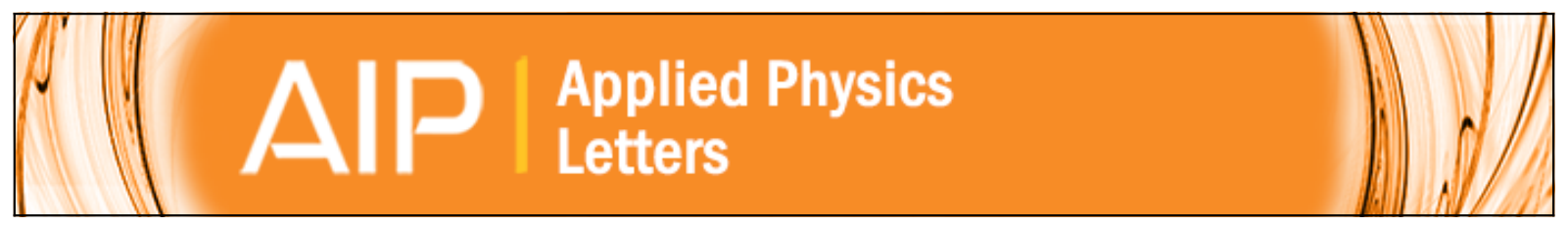

Photodetectors fabricated from a self-assembly of a deoxyguanosine derivative

R. Rinaldi, E. Branca, R. Cingolani, S. Masiero, G. P. Spada, and G. Gottarelli

Citation: Applied Physics Letters 78, 3541 (2001); doi: 10.1063/1.1374232

View online: http://dx.doi.org/10.1063/1.1374232

View Table of Contents: http://scitation.aip.org/content/aip/journal/apl/78/22?ver=pdfcov

Published by the AIP Publishing

Articles you may be interested in

Self-assembled deoxyguanosine based molecular electronic device on $\mathrm{GaN}$ substrates

Appl. Phys. Lett. 92, 013309 (2008); 10.1063/1.2828405

Fabrication of DNA Mediated Devices: Alignment of single DNA and the 1D pattern of DNA selfassembly AIP Conf. Proc. 859, 3 (2006); 10.1063/1.2360581

Selfassembled DNA Structures for Nanoconstruction

AIP Conf. Proc. 725, 43 (2004); 10.1063/1.1805377

SelfAssembly Experiments with PNADerivatized Carbon Nanotubes

AIP Conf. Proc. 725, 25 (2004); 10.1063/1.1805375

Self-assembled DNA networks and their electrical conductivity

Appl. Phys. Lett. 77, 3105 (2000); 10.1063/1.1323546 


\title{
Photodetectors fabricated from a self-assembly of a deoxyguanosine derivative
}

\author{
R. Rinaldi, a) E. Branca, and R. Cingolani \\ National Nanotechnology Laboratory (NNL)-Istituto Nazionale per la Fisica della Materia (INFM), and \\ Dipartimento di Ingegneria dell'Innovazione, Università di Lecce, Italy
}

S. Masiero, G. P. Spada, and G. Gottarelli

Dipartimento di Chimica Organica, “A. Mangini," Università di Bologna, Italy

(Received 12 January 2001; accepted for publication 29 March 2001)

\begin{abstract}
A metal-semiconductor-metal (MSM) photodetector has been fabricated using as the semiconductor, a self-assembled layer of a DNA basis, namely a deoxyguanosine derivative, deposited between two gold electrodes. These were defined lithographically on a $\mathrm{SiO}_{2}$ substrate, separated by a distance of about $120 \mathrm{~nm}$. The resulting self-assembled guanosine crystal has been deposited in such a way to achieve striking semiconducting properties. We show that with these conditions, the $I-V$ characteristics are independent of the crystal orientation. The device shows a high current response (differential resistance at room temperature ranges in $M \Omega$ ) which is symmetric with respect to bias sign and dependent on the illumination conditions. This behavior can be explained by taking into account the standard MSM theory and its applications as a photodetector. (C) 2001 American Institute of Physics. [DOI: 10.1063/1.1374232]
\end{abstract}

Observations of efficient charge transport in biological materials, such as DNA segments, have stimulated much research on the physical mechanism responsible for the conduction $^{1-9}$ and on its possible applications. ${ }^{10}$ Recent interest in molecular-scale electronics as a means to fabricate extremely dense logic and memory circuits has led to the development of molecular diodes, switches, ${ }^{10}$ photodetectors and rectifier devices. ${ }^{11-13}$ The current-voltage characteristics of a metal-semiconductor-metal photodetector (essentially two Schottky diodes connected back to back) are well known ${ }^{14,15}$ and have been studied on the basis of the thermionic emission and tunneling theory (the latter when the semiconductor length is small enough to permit tunneling). In this work we present a hybrid photodetector, in which the uniformly doped semiconductor has been replaced by selfassembled layers of a deoxyguanosine derivative.

A pattern of two electrodes on a PMMA photoresist deposited on a $\mathrm{SiO}_{2}$ substrate was fabricated by electron beam lithography (EBL) and, by lift-off processing, layers of chromium $(\cong 8 \mathrm{~nm})$ and gold $(\cong 30 \mathrm{~nm})$ were used to implement the device. This consists of two electrodes of width $10 \mu \mathrm{m}$, length $5 \mathrm{~mm}$ and with arrow shaped facing edges. The distance $L$ between the two electrodes was varied in the range $30<L<150 \mathrm{~nm}$. A schematic of the device is shown in Fig. 1(a) together with the energy band diagram of the MSM device at the equilibrium [Fig. 1(b)]. In this letter we focus on devices with spacing of the order of $L=100 \mathrm{~nm}$, which is comparable to the self-organizing length of our molecules. The biological semiconductor was obtained from a solution of deoxyguanosine derivative in chloroform $\left(3.3 \times 10^{-2} \mathrm{M}\right)$. This liquid was deposited on the device by a Hamilton syringe-the volume of each drop was $\cong 3 \mu$ l (corresponding to $10^{5}$ molecules in the spacing region). After having waited until the solvent had evaporated, atomic force microscope

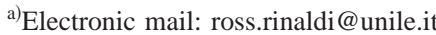

(AFM) measurements (in contact mode) of the morphological structure of the film were taken. From these measurements (Fig. 2), it is possible to see, on the atomic scale, details of the self-assembly ${ }^{16}$ of this biological material in the form of ribbons: this feature is characteristic of this particular concentration of solution. The morphology of this "crystal" is that of an array of chains of molecules linked in the $\mathrm{H}$-acceptor and $\mathrm{H}$-donor sites (oxygen and nitrogen atoms on one side and $\mathrm{NH}$ and $\mathrm{NH}_{2}$ groups in the other side, as shown in Fig. 2). The superposition of the chemical scheme with AFM images demonstrates the self-assembly of this

(a)

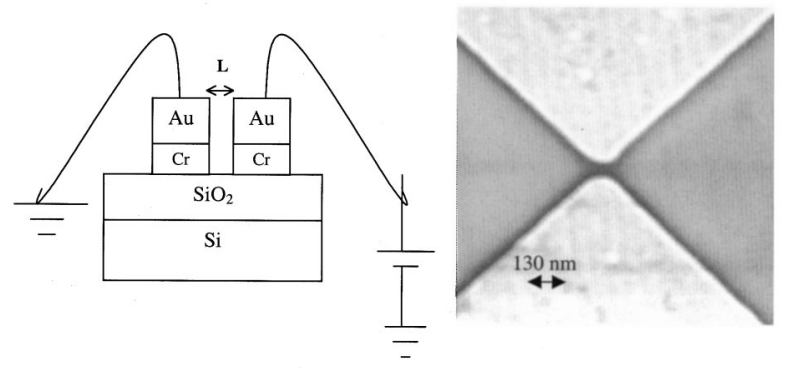

(b)

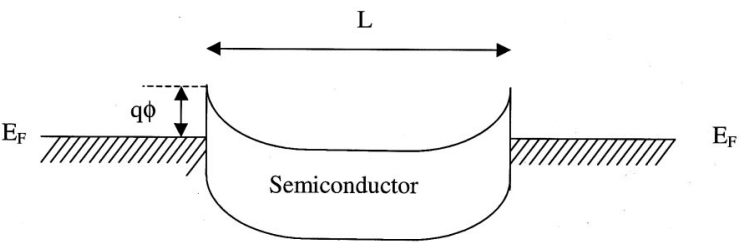

FIG. 1. (a) Section of the device used in the experiment. The next image is a SEM image of the gold nanoelectrodes fabricated by EBL and lift-off onto a $\mathrm{SiO}_{2} / \mathrm{Si}$ substrate. (b) Band diagram of the MSM device at the thermodynamic equilibrium. 

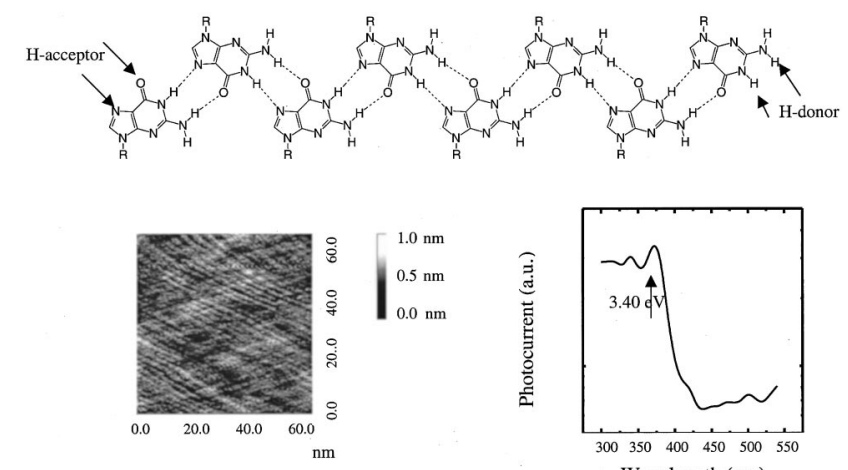

FIG. 2. Schematics of the ribbon-like structure formed by the deoxyguanosine molecules connected through hydrogen bonds (top). Here $R$ is a radical containing the sugar and alkyl chains. AFM micrograph (bottom, left) of the ordered solid state deoxyguanosine film obtained after drying one drop of the solution in the gate (with concentration of $\sim 3.3 \times 10^{-2} \mathrm{M}$ ). The regular arrangement of ribbons ranges over a distance of about $100 \mathrm{~nm}$. The ribbon width of about $3 \mathrm{~nm}$ is consistent with that determined by $\mathrm{x}$-ray measurements. The AFM measurements were performed with atomic resolution in contact mode on the top surface of the film. Photocurrent diagram (bottom, right) obtained for the device (biased by $V=1 \mathrm{~V}$ ).

material in the form of aligned biological wires. In a previous report we have shown that the electrical-transport properties are periodical as the morphology, so that the crystals of guanosine can be described in the frame of a band-like transport model. Extensive transport and photocurrent experiments, performed on devices with different contact spacing $30<L<1000 \mathrm{~nm}$, together with density functional calculations, assuming the molecular ordering obtained by the AFM, have revealed that the self-assembled deoxyguanosine films behave like wide gap semiconductors, with energy gap in the range of $3-3.5 \mathrm{eV}$ and electron effective mass $m_{e}$ $\cong 2 \mathrm{~m}_{0} \cdot{ }^{17}$ This is clearly shown in Fig. 2, where we display the photocurrent spectrum taken at room temperature from the device of Fig. 1(a). An evident energy gap onset ( 3.4 $\mathrm{eV}$ ) proves the band-like description of this particular biological material (at this concentration), and its similarities to other wide-band-gap materials, such as GaN, with near UV absorption onset. The ordering conditions of the DG (deoxyguanosine) film are essential for the current response of the device. If a more or less diluted solution is used, selfassembly does not occur; therefore great care has to be taken in order to control the solid state assembly of the molecules. $I-V$ measurements were taken using a picoammeter (Keithley 617) both in dark and under illumination. Figure 3 shows the experimental $I-V$ measurements taken for a device with a $120 \mathrm{~nm}$ gap and one drop of material ${ }^{18}$ in dark and under illumination (inset of Fig. 3).

The observed characteristics are typical of a MSM system. A MSM device has two Schottky barriers connected back to back: bias of any polarity will put one Schottky barrier in the reverse direction and the other in the forward direction. Because of the small width of this "semiconductor," it easily becomes depleted and the dark current in the forward biased junction is not the usual high electron forward bias current. Instead, the dark current in the forward biased junction is due to the hole and electrons currents injected from the metal over the barrier.

The $I-V$ characteristics of a MSM device thus consists of two distinct regions: a steep rise around $V=0$, and a satu-

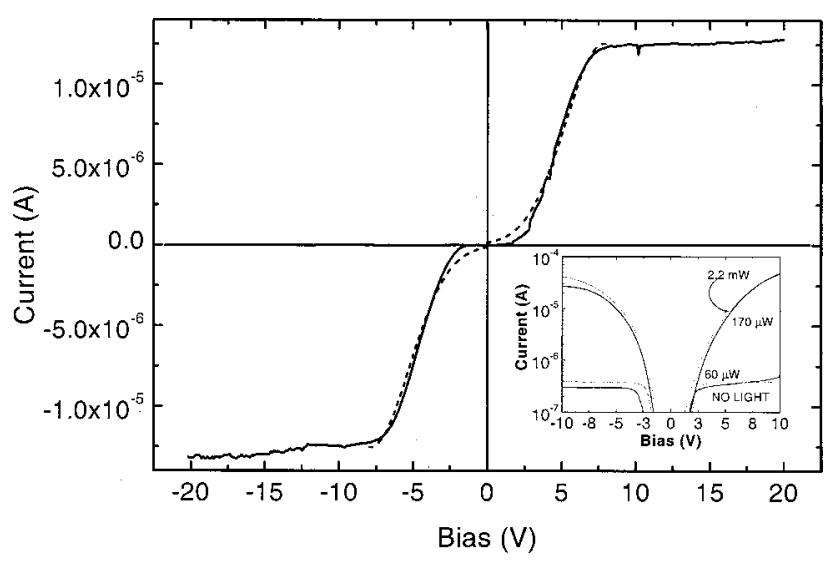

FIG. 3. $I-V$ characteristics of the MSM device (dark current): the continuous line represents the experimental data while the dashed line is the fit of Eq. (1) in the steep rise region. In the inset several curves are shown at different power illuminations conditions.

ration region for high bias. The steep rise around $V=0$ is described by:

$$
I=I_{s} e^{-\beta\left(V-V_{\mathrm{FB}}\right)^{2} / V_{\mathrm{FB}}}
$$

where $I_{s}$ is a constant, $\beta=e / 4 K_{B} T$ and $V_{\mathrm{FB}}$ is the flat-band voltage with which the electrical field in $x=L$ is 0 . The saturation region of the device is given by the reverse saturation currents from electrons and holes:

$$
J=A_{n}^{*} T^{2} e_{b n}^{-e \phi / k T}+A_{p}^{*} T^{2} e_{p n}^{-e \phi / k T}
$$

where $A_{n}, A_{p}$ are the electron and the hole effective Richardson constants, $\phi_{b n}, \phi_{g p}$ are the Schottky barriers. Upon saturation the current response reaches values of the order of tens of $\mu \mathrm{A}$, which corresponds to differential resistance values of the order of a fraction of a $\mathrm{M} \Omega$. From the geometry of the system it is possible to infer that the resistivity $(\rho)$ of this material is around $4 \Omega \mathrm{cm}$. The voltage at which the photocurrent saturates corresponds to the flat-band condition, in which the electric field at the anode becomes zero. This can be estimated from a one-dimensional depletion equation:

$$
V_{\mathrm{FB}}=\left(\frac{e N}{2 \epsilon_{s}}\right) L^{2},
$$

where $N$ is the ionized impurity concentration, $L$ is the semiconductor width, and $\epsilon_{s}$ is the dielectric constant of the semiconductor. Figure 3 shows the results of a simulation of the MSM photodetector characteristics from Eq. (1), in the nonsaturated region of the $I-V$ graph (dashed lines), compared to the experimental measurements. The agreement is quite good, providing a $V_{\mathrm{FB}}$ value of about $7.9 \mathrm{~V}$. The applicability of the MSM theory to this situation is a further proof to the semiconductor behavior of the self-assembled deoxyguanosine film. The non-ohmic-behavior is also dependent on the illumination conditions: the response shows a saturation current that depends on the wavelength and intensity of the incident flux. When black-body light is received in the gap between the two gold contacts, the photocurrent at first rises with voltage, then becomes saturated at current values which depend on the illumination powers. In the inset of Fig. 3 different saturation currents are observed with respect to different black body illumination powers. This could be due to incoherent transport which is not dependent on the wave- 
length of the incoming photon, but on the intensity of the incident flux. The response of this device to varying powers of the black-body illumination can reach values as high as $\cong 1 \mathrm{~A} / \mathrm{W}$, indicating the high gain of the photoconductive detector. The width of the semiconducting material is comparable to the Debye length, at least for higher electric fields, and the device is completely depleted (MOTT barrier). Under these conditions, the structure exhibits good photodetection behavior like a fast photoconductive detector.

Finally, we would like to briefly comment on the transport mechanism across the DG film. Charge transport in our devices does not occur directly through the molecular wires, but conductivity is a band-like phenomenon involving the crystal of DG as a whole. In fact from the AFM measurements, made on the analyzed devices, it is possible to argue that the orientation of the single ribbon with respect to the device axis does not influence the transport. The order condition is the only condition necessary for the achievement of high current response. This can be interpreted as a requirement for a sequential succession of the molecules, but not as a condition for a charge transfer through the individual onedimensional ribbon.

In conclusion, a photodetector has been fabricated from deoxyguanosine derivative. This device shows high responsivity $(1 \mathrm{~A} / \mathrm{W})$ and, from the experimental data, an equivalent semiconductor energy gap of about $3.4 \mathrm{eV}$ at room temperature is observed. The $I-V$ characteristics show a symmetric behavior that can be explained by the contributions of the band-like transport of the guanosine as semiconductor. Provided the self-organization length can be increased up to $250 \mathrm{~nm}$, one could conceive a new generation of biomolecular planar devices, written by an ink-jet printer onto standard lithographically defined patterns.
${ }^{1}$ K. Fukui and K. Tanaka, Angew. Chem. Int. Ed. Engl. 37, 158 (1998).

${ }^{2}$ C. J. Murphy, M. R. Arkin, Y. Jenkins, N. D. Ghatlia, S. H. Brossmann, N. J. Turro, and J. K. Burton, Science 262, 1025 (1993).

${ }^{3}$ M. R. Arkin, E. D. A. Stemp, R. E. Holmlin, J. K. Barton, A. Hörmann, E. J. C. Olson, and P. F. Barbara, Science 273, 475 (1996).

${ }^{4}$ B. Hall, R. Erik Holmin, and J. K. Barton, Nature (London) 382, 731 (1996).

${ }^{5}$ F. Lewis, T. Wu, Y. Zhang, R. L. Letsinger, S. R. Greenfield, and M. R. Wasielewski, Science 277, 673 (1997).

${ }^{6}$ E. Meggers, D. Kusch, M. Spichty, U. Wille, and B. Geise, Angew. Chem. Int. Ed. Engl. 37, 460 (1998).

${ }^{7}$ B. Giese, S. Wessely, M. Spormann, U. Lindemann, E. Meggers, and M. E. Michel-Beyerle, Angew. Chem. Int. Ed. Engl. 38, 996 (1999).

${ }^{8}$ A. Harriman, Angew. Chem. Int. Ed. Engl. 38, 945 (1999).

${ }^{9}$ Y. Okahata, T. Kobayashi, K. Tanaka, and M. Shimomura, J. Am. Chem. Soc. 120, 6165 (1998).

${ }^{10}$ D. M. Eigler, C. P. Lutz, and W. E. Rudge, Nature (London) 352, 600 (1991).

${ }^{11}$ R. Rinaldi, E. Branca, R. Cingolani, R. De Felice, E. Molinari, S. Masiero, G. P. Spada, G. Gottarelli, and A. Garbesi (unpublished).

${ }^{12}$ A. S. Martin and J. R. Sambles, Phys. Rev. Lett. 70, 218 (1993).

${ }^{13}$ A. Aviram and M. A. Ratner, Chem. Phys. Lett. 29, 277 (1974).

${ }^{14}$ K. K. Ng, Complete Guide to Semiconductor Devices (McGraw-Hill International Editions, New York, 1995).

${ }^{15}$ S. M. Sze, D. J. Coleman, J. R. Loya, and A. Loya, Solid-State Electron. 14, 1209 (1971).

${ }^{16}$ G. Gottarelli, S. Masiero, E. Mezzina, G. Spada, P. Mariani, and M. Recanatini, Helv. Chim. Acta 81, 2078 (1998).

${ }^{17}$ R. Di Felice, E. Molinari, R. Rinaldi, E. Branca, R. Cingolani, A. Garbesi, S. Masiero, G. P. Spada, and G. Gottarelli, Proceedings of the 25th International Conference on the Physics of Semiconductors (World Scientific, Singapore, 2001), Vol. 279.

${ }^{18}$ Similar data were taken for different concentrations and gap lengths. The results show that with decreasing gap length the $I-V$ characteristics are more asymmetric and that with concentrations very different (one order of magnitude more or less) from $3.3 \times 10^{-2} \mathrm{M}$, the current response is quite close to zero. 